\title{
Managing Things and Services with Semantics: A Survey
}

\author{
Matthias Thoma* ${ }^{*}$ Torsten Braun ${ }^{\ddagger}$ Carsten Magerkurth* Alexandru-Florian Antonescu* ${ }^{* \ddagger}$ \\ ${ }^{*}$ SAP (Switzerland) Inc., Althardstrasse 80, 8105 Regensdorf, Switzerland \\ ${ }^{\ddagger}$ Communication and Distributed Systems, University of Bern, Neubrückstrasse 10, 3012 Bern, Switzerland \\ matthias.thoma@sap.com, braun@iam.unibe.ch, carsten.magerkurth@sap.com, alexandru-florian.antonescu@sap.com
}

\begin{abstract}
This paper presents a survey on the usage, opportunities and pitfalls of semantic technologies in the Internet of Things. The survey was conducted in the context of a semantic enterprise integration platform. In total we surveyed sixty-one individuals from industry and academia on their views and current usage of IoT technologies in general, and semantic technologies in particular. Our semantic enterprise integration platform aims for interoperability at a service level, as well as at a protocol level. Therefore, also questions regarding the use of application layer protocols, network layer protocols and management protocols were integrated into the survey. The survey suggests that there is still a lot of heterogeneity in IoT technologies, but first indications of the use of standardized protocols exist. Semantic technologies are being recognized as of potential use, mainly in the management of things and services. Nonetheless, the participants still see many obstacles which hinder the widespread use of semantic technologies: Firstly, a lack of training as traditional embedded programmers are not well aware of semantic technologies. Secondly, a lack of standardization in ontologies, which would enable interoperability and thirdly, a lack of good tooling support.
\end{abstract}

\section{INTRODUCTION}

Research on semantics and semantic management of Internet of Things systems has attracted a lot of interest in the last ten years, but so far, has failed to gain widespread use in industrial applications. From an European research point of view (but not limited to that) a lot of resources, both in manpower as well as in financial support, has gone into semantic research. Most projects that have been ramped up in past five years in the context of Future Internet [1], Internet of Things [2] or Industry 4.0 [3] use semantic technologies in one way or the other. Just to name a few, the SENSEI project [4] for example, was funded with $€ 14.9$ million. The more recent Internet-of-Things Architecture project (IOT-A) [5], which is considered as an EU flagship project, received around $€ 11.9$ million. More semantic IoT-related projects will start as part of HORIZON 2020. So there is definitely a lot of research being conducted, but when looking into commercialized products it is obvious that semantic technologies in IoT so far failed to deliver on its promise. Semantic technologies still are not of wide-spread use in real-world applications.

In this work, we present a recent study on semantics in Internet-of-Things applications, that was mainly conducted to gain insight into potential further usage cases of semantic platforms and further development options. Our work on linked services [6] [7] motivated surveying transport and application layer protocols, which otherwise is not much connected with semantic technologies. While the main focus of the survey was to get a feeling about the view of the community on semantics, it also revealed some interesting insights about application level protocols, transport level protocols and network management which is of interest for a broader audience.

\section{SURVEY}

\section{A. Introduction}

We conducted a survey on semantics with an emphasis on integration of enterprise IT systems. Semantics are currently under intense research from both industry and academia. Our objective was to identify the actual needs of IoT with regard to semantic support and to identify current shortcomings.

\section{B. Methodology}

The survey was distributed among experts, from both industry (among others: SAP, IBM, NEC, Orange, Telefonica) and academia. While some experts were recruited directly, the majority of the respondents were self-selected. They survey was conducted online and anonymity was guaranteed and technically enforced by the system. As IoT is a very broad field, we explicitly excluded all kinds of mobile phone development and limited protocol related questions specifically to systems where an ISO/OSI-like stack is being used. Nonetheless, we also briefly surveyed the usage of other technologies.

\section{Threats to internal or external validity}

As the study was conducted anonymously it is not possible to validate that the claims made are valid. We added sanity checks that allowed to filter non valid responses. No incentives were given for participating. Most industrial participants worked with IoT-systems in industrial automation, retail or logistics. The responses by participants from academia were (if a sector was chosen) mainly from automation and logistics, and the broad areas of smart city. Other sub-fields of IoT may have different requirements regarding protocols, but we expect the tendencies discovered in our survey to be generalizable.

\section{Results}

In the following we present and discuss the results of the survey. We categorize the results into four groups: (i) General statistical information about the participants and their skillsets, (ii) Protocols, (iii) Semantics and (iv) Enterprise Integration. 
1) General: The total number of participants was sixtyone. Their experience levels, origin and skillsets are detailed out in Table I. There were nearly as many participants from industry as from academia. The majority of participants had at least three years of professional experience and a more than basic understanding of IoT and semantics. Most people from academia had experience (skills) on the advanced or expert level. Naturally, the expertness in enterprise software and systems was higher for the industry participants.

TABLE I

PARTICIPANT GROUP: EXPERIENCE AND SKILLS

\begin{tabular}{|c|c|c|c|}
\hline \multicolumn{2}{|l|}{ Participants } & \multicolumn{2}{|l|}{ Career level } \\
\hline Total & 61 & Entry & 8 \\
\hline Industry & 32 & Advanced & 17 \\
\hline Academia & 29 & Professional & 36 \\
\hline \multicolumn{2}{|l|}{ Experience (in years) } & \multicolumn{2}{|l|}{ Skills (IoT) } \\
\hline $1-2$ & 6 & No experience & 0 \\
\hline $3-5$ & 31 & Beginner & 11 \\
\hline $5-9$ & 15 & Some experience & 22 \\
\hline $10-14$ & 5 & Advanced & 21 \\
\hline$>15$ & 4 & Expert & 7 \\
\hline \multicolumn{2}{|l|}{ Skills (Semantics) } & \multicolumn{2}{|l|}{ Skills (Enterprise systems) } \\
\hline Beginner & 26 & Beginner & 28 \\
\hline Some experience & 12 & Some experience & 11 \\
\hline Advanced & 17 & Advanced & 14 \\
\hline Expert & 6 & Expert & 8 \\
\hline \multicolumn{4}{|l|}{ Sector } \\
\hline Industrial automation & 14 & Home automation & 4 \\
\hline Retail & 12 & Transportation and logistics & 7 \\
\hline Smart City & 5 & Healthcare & 2 \\
\hline Vehicular communications & 5 & Other / none & 12 \\
\hline
\end{tabular}

Most of the projects were either in the area of (wireless) sensor network or other connected (embedded) constrained devices. While the actual devices were almost always constrained, the network was not. There was a large group using 802.15.4 based wireless, but also some with 802.11 networks, a regular (Ethernet) wired connection or combinations thereof.

2) Protocols: Application layer protocols, as shown in Table II, seem still to be dominated by custom written protocols. Nonetheless, standardized protocols like HTTP, CoAP[8] or MQTT[9] are used by nearly half of the participants. SOAP, which is otherwise widely used in enterprises [10], does not seem to play a role at all. While not adopted widespread yet, in future, most people seem to anticipate CoAP as one of the major players, winning shares from all other standardized protocols and the custom ones. Nonetheless, when comparing the protocols planned to be used in future own developments (i. e. what the participants really plan) and the expected future usage of the industry as a whole (i. e. what they think the industry will move towards), than the expectations towards CoAP are even higher. The number of people planning with and expecting custom protocols is still quite high. In terms of network/transport layer protocols there seems to be an expected shift towards IPv6/6LoWPAN and UDP/CoAP based protocols. It is surprising, given the size of the ZigBee Alliance, that the ZigBee protocol suite is not used more often.
ZigBee was almost always selected together with 6LoWPAN, so that most likely even within the ZigBee universe its IPv6 enhancements (Zigbee IP) are used to ensure interoperability.

TABLE II

USED PROTOCOLS

\begin{tabular}{|c|c|c|c|}
\hline Application layer & Now & $\begin{array}{l}\text { Future } \\
\text { (planned) }\end{array}$ & $\begin{array}{l}\text { Future } \\
\text { (expected) }\end{array}$ \\
\hline CoAP & $8 \%$ & $28 \%$ & $44 \%$ \\
\hline HTTP & $14 \%$ & $12 \%$ & $21 \%$ \\
\hline SOAP & $2 \%$ & $2 \%$ & $6 \%$ \\
\hline CAN & $2 \%$ & $4 \%$ & $4 \%$ \\
\hline MQTT & $5 \%$ & $6 \%$ & $7 \%$ \\
\hline KNX & $6 \%$ & $6 \%$ & $7 \%$ \\
\hline MODBUS & $4 \%$ & $6 \%$ & $7 \%$ \\
\hline Other/Custom & $47 \%$ & $38 \%$ & $10 \%$ \\
\hline Zigbee & $8 \%$ & $10 \%$ & $8 \%$ \\
\hline Transport layer & Now & Planned & Expected \\
\hline UDP & $19 \%$ & $21 \%$ & $14 \%$ \\
\hline Reliable UDP (non CoAP) & $24 \%$ & $23 \%$ & $12 \%$ \\
\hline TCP & $13 \%$ & $11 \%$ & $9 \%$ \\
\hline $\mathrm{UDP}+\mathrm{CoAP}$ & $8 \%$ & $24 \%$ & $39 \%$ \\
\hline Custom/other (TCP-like) & $13 \%$ & $7 \%$ & $14 \%$ \\
\hline Custom/other & $23 \%$ & $14 \%$ & $12 \%$ \\
\hline Network layer & Now & Planned & Expected \\
\hline IPv4 & $5 \%$ & $3 \%$ & $2 \%$ \\
\hline IPv6 & $21 \%$ & $23 \%$ & $35 \%$ \\
\hline 6LoWPAN & $25 \%$ & $45 \%$ & $32 \%$ \\
\hline Custom 802.15.4 protocol & $21 \%$ & $14 \%$ & $10 \%$ \\
\hline Custom (other) & $22 \%$ & $9 \%$ & $12 \%$ \\
\hline Zigbee & $6 \%$ & $6 \%$ & $9 \%$ \\
\hline (Dedicated) Network Management & Now & Planned & Expected \\
\hline CMIP/CMIS & $4 \%$ & $3 \%$ & $5 \%$ \\
\hline SNMP & $15 \%$ & $24 \%$ & $49 \%$ \\
\hline IEC104 & $0 \%$ & $2 \%$ & $8 \%$ \\
\hline $\begin{array}{l}\text { Custom protocol based on } \\
\text { application layer protocol }\end{array}$ & $44 \%$ & $37 \%$ & $20 \%$ \\
\hline Other custom protocol & $37 \%$ & $34 \%$ & $18 \%$ \\
\hline None / not at all & $24 \%$ & $18 \%$ & $4 \%$ \\
\hline
\end{tabular}

The views on the community with regards to the current IoT-protocols has been surveyed with a 4-point Likert-style questionnaire. The Likert items as well as the responses are illustrated in Figure 1. There seems to be consensus that most future IoT-applications will be IP-based to some degree, and a bias towards ReST-based architectures. This matches the results in Table II.

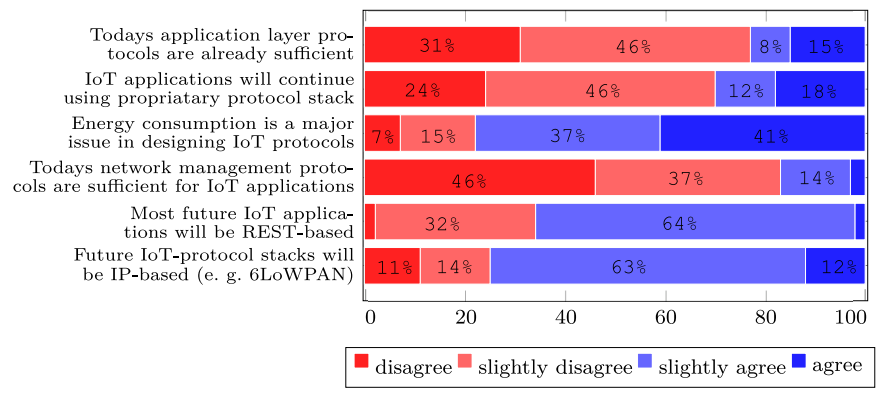

Fig. 1. Usage and potential of IoT-protocols (on a 4-point Likert scale)

Dedicated management protocols, like SNMP[11] or 
TABLE III

TECHNOLOGIES USED (IN PERCENT OF PARTICIPANTS), MULTIPLE ANSWERS WERE POSSIBLE

\begin{tabular}{llll}
\hline Technology & Now & $\begin{array}{l}\text { Future } \\
\text { (planned) }\end{array}$ & $\begin{array}{l}\text { Future } \\
\text { (expected) }\end{array}$ \\
\hline RFID & $25 \%$ & $27 \%$ & $24 \%$ \\
QR codes & $5 \%$ & $8 \%$ & $11 \%$ \\
Barcodes & $12 \%$ & $10 \%$ & $7 \%$ \\
NFC & $10 \%$ & $15 \%$ & $39 \%$ \\
Bluetooth & $7 \%$ & $6 \%$ & $19 \%$ \\
\hline None & $41 \%$ & $34 \%$ & $-\%$ \\
\hline
\end{tabular}

CMIP/CMIS[12], seem to be not that widely used at the moment as one would expect. Most participants, used (if at all), a custom application-specific layer on top of the already used application layer protocol. Most participants $(>70 \%)$ consider nowadays management protocols as not sufficient enough (Figure 1). Interestingly, many respondents also do not plan to use a standardized protocol in future. Generally speaking, the need for management seems to be recognized though, as a more widespread use is expected in future. When asked why not using an existing management protocol, the vast majority answered that they expected the overhead of a standardized protocol as too high, or that they fear negative consequences with regard to performance and power consumption. The usage of a custom protocol, tailored towards their specific needs, seems to give them more confidence in the qualitative and quantitative properties of the system, even for the price of a lack of interoperability. As these concerns are not new, there are efforts to run (subsets) of, for example, SNMP [13][14] and NETCONF [15] on resource constrained nodes. CoAP-based protocols (e.g. [16]) have also been investigated, nonetheless, these are also non standardized custom protocols on top of the application layer protocol.

Considering that the IoT to some degree originated from RFID, we surveyed other technologies than those based on ISO/OSI (Internet)-like stacks. As can be seen in Table III quite some people use RFID, Bluetooth and QR codes. NFC still seems not that much used at the moment by the participants. Nonetheless, this might be due to the participants as none of them was from financial sector or mobile ticketing, where NFC has gained some usage. Those who are using NFC are solely from retail and industrial automation.

3) Semantics: The general attitude of the community towards semantics was surveyed with a four point Likert-style questionnaire. The individual Likert items and the distribution of the answers are shown in Figure 2. Most participants agree that semantics will play a role in future IoT systems. Some nonetheless, think that it is too bloated/an academic toy, or as one of the participants wrote "a hype from bored academics that noone will remember in a few years" When asked what is needed for a widespread adoption of semantics in the IoT (see Figure 3) the by-far most often mentioned issues were knowledge / awareness of development staff and standardization, followed by development tool support. Infrastructure and tool support for domain experts did not seem to be an issue. As illustrated in Figure 4 the main

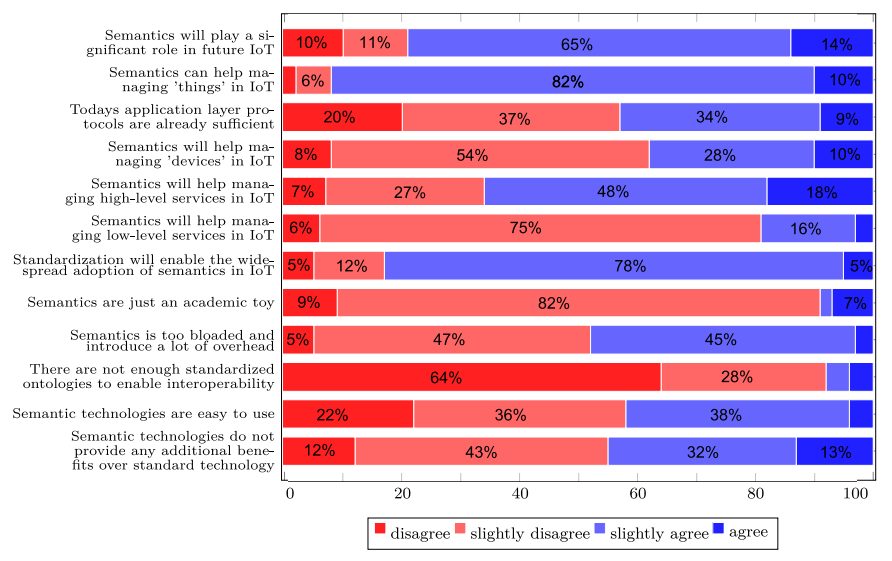

Fig. 2. Usage and potential of semantics (on a 4-point Likert scale)

advantages of semantics is seen in high-level interoperability and the management of things, followed by reasoning and the management of devices. Interoperability at an endpoint level, as suggested in our integration platform, is not yet seen as an area where semantics can contribute.

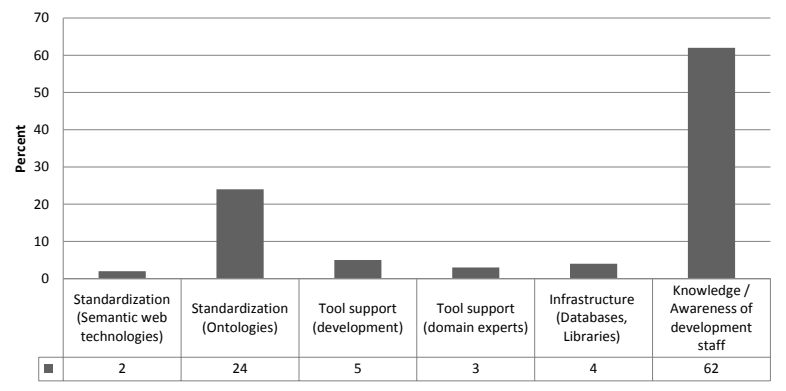

Fig. 3. Main obstacle for not using semantic technologies (in percent)

Given the fact that most people think that there is potential in semantics, it is interesting to see its actual usage: As shown in Table IV, out of all participants $41 \%$ do not use semantics at all and $34 \%$ do not plan to use it in a further project. From those who use semantics the vast majority is using it for the description of things, devices or services. Reasoning on top of the semantic data seems to be a topic that many people have on their radar. When asked which (domain specific) ontologies are used, most answers centered around custom/problem specific ontologies. The only ontology that was mentioned more often was SSN. The lack of standardization or at least of de-facto standards has been mentioned several times.

TABLE IV

SEMANTIC TECHNOLOGY USAGE (PER PARTICPANTS NOT SELECTING NONE), MULTIPLE ANSWERS WERE POSSIBLE

\begin{tabular}{lll}
\hline Technology & Now & $\begin{array}{l}\text { Future } \\
\text { (planned) }\end{array}$ \\
\hline Description of endpoint level services & $7 \%$ & $22 \%$ \\
Description of Things & $67 \%$ & $78 \%$ \\
Description of Devices & $32 \%$ & $48 \%$ \\
Description of high-level services & $38 \%$ & $45 \%$ \\
Reasoning & $22 \%$ & $35 \%$ \\
Configuration & $5 \%$ & $12 \%$ \\
\hline None / Not at all & $41 \%$ & $34 \%$ \\
\hline
\end{tabular}


The time horizon of supporting semantics in a product or product prototype (industry), or do research is shown in Table V. Industry participants generally expected to not do or use semantics in the next 2 years and more $(>70 \%)$. Participants from academia do plan to work with semantics often and within a timespan of less than two years (also around 70\%).

TABLE V

USE OF SEMANTIC TECHNOLOGIES (TIME HORIZON)

\begin{tabular}{lll}
\hline & Industry & Research \\
\hline not at all & $5 \%$ & $12 \%$ \\
next 6 months & $8 \%$ & $24 \%$ \\
1 year & $11 \%$ & $35 \%$ \\
2 years & $24 \%$ & $17 \%$ \\
more than 2 years & $52 \%$ & $12 \%$ \\
\hline
\end{tabular}

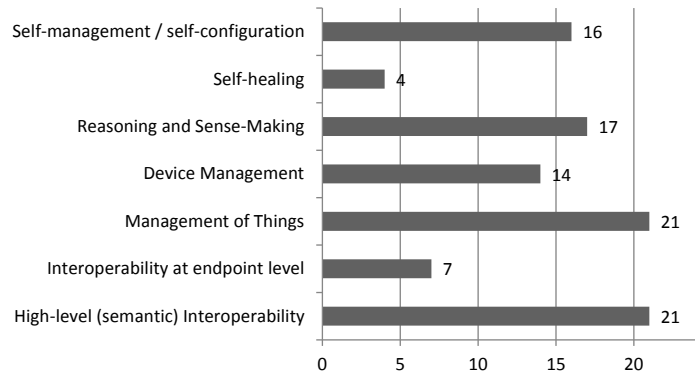

Fig. 4. Attitude towards opportunities/usages of semantics in IoT

4) Enterprise Integration: Enterprise integration is still mainly done through middleware or proxies, in cases where appropriate. Direct communication, without an intermediary, has not yet been widely adopted in enterprise integration frameworks. As shown in Figure 5, only 17\% of the integration projects were done via a direct access (e. g. IPv6), while most solutions seem still to use a gateway. This is most likely due to historical reasons, as IoT used to use custom protocols which required a gateway solution. Most IoT-systems run by our participants have either a fully automated configuration scheme or some kind of technical administrator responsible. End-users are only in 14\% of all cases solely responsible for the configuration of their device. Most monitoring and management (Figure 6) activities in such environments are also done on this intermediary. Data gathering and aggregation is mostly done either solely on the device $(37 \%)$ or on the gateway (46\%). This, to some degree, might also explain why management protocols did not gain widespread use yet. Most monitoring platforms are centralized, and often do not yet provide a real-time view on the system. A device-level only monitoring (e. g. by the user only) is not very common.

\section{CONCLUSION}

While the survey is only a still picture of a subset of the current situation in IoT, some conclusions can be drawn. The IoT domain remains to be highly heterogeneous. While semantics are expected to play a role in future IoT systems, there is still a way to go. Most participants see some benefit in the semantic management of things, devices and services.

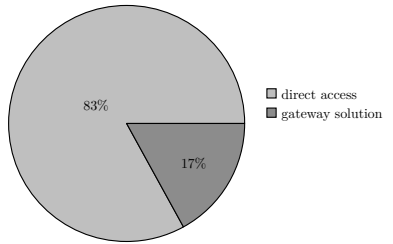

(a) Direct access on network/transport layer vs gateway solutions

Fig. 5. Enterprise integration

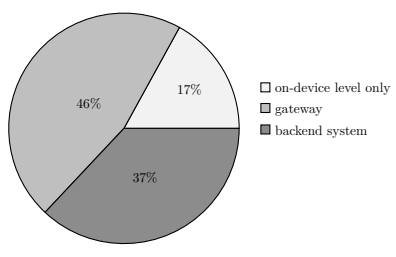

(a) Data gathering/aggregation

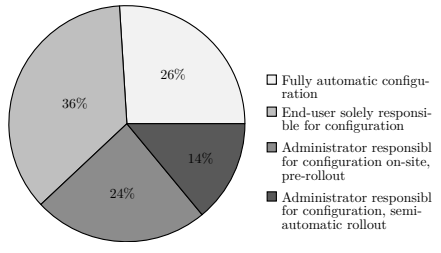

(b) Configuration
Fig. 6. Monitoring

Nonetheless, when looking into the actual situation and the planned usage of semantics in IoT, these benefits seem not to be strong enough to stimulate large scale usage. One reason could be a lack of training in semantics and the more "bit and byte"-oriented skillset current embedded developers have.

It is obvious that semantic management of services needs to take different protocols into account, even if there seems to be the expectation that IPv6/6LoWPAN will play a crucial role and finally make the IoT vision a reality. CoAP, while currently not used at a large scale, is expected to be for IoT what was HTTP for the WWW. Nonetheless, the number of people using or expecting the use of custom protocols in the future is quite high. Gateways or proxies will still be widely used. It seems as if a convergence towards an internet standard might not happen as soon as expected. Semantic service descriptions could fill that gap and allow integration of different protocols. SSN seems to have emerged to a de-facto standard for research in sensor networks. It was also one of the very few mature enough choices available when we sketched our own integration platform, a view that seems to be shared by others. The management of things and corresponding functionality (like discovery) seemed most promising to the participants of our study, followed by devices and services. Here, in our opinion, the community has still a way ahead before it stands on a common ground.

Our survey is only one small step towards a qualitative and quantitative understanding of real-world usage in the emerging field of the IoT. Not only in terms of the use of semantics, but also in the use of protocols and the management of things, services and devices. The authors suggest further empiric work to broaden the databasis and deepen the understanding of used protocols, needed management functionality and problems arising in real IoT deployments. Especially, the first industrial deployments of semantic platforms will lead to further insights into the real problems arising when using semantic technologies and if semantics can really hold its promises. 


\section{REFERENCES}

[1] "European Future Internet Portal." [Online]. Available: http://www.future-internet.eu/

[2] N. Gershenfeld, R. Krikorian, and D. Cohen, "The Internet of things." Scientific American, vol. 291, no. 4, p. 76, 2004.

[3] W. Dangelmaier, C. Laroque, and A. Klaas, "Industrie 4.0Handlungsfelder der Digitalen Fabrik zur Optimierung der Ressourceneffizienz in der Produktion."

[4] "Sensei - Integrating the Physical with the Digital World of the Network of the Future." [Online]. Available: http://www.sensei-project.eu/

[5] "Internet of Things Architecture (IOT-A)." [Online]. Available: http://www.iot-a.eu/public

[6] M. Thoma, K. Sperner, T. Braun, and C. Magerkurth, "Integration of WSNs into enterprise systems based on semantic physical business entities," in Wireless Days (WD), 2013 IFIP, 2013.

[7] M. Thoma, T. Braun, and C. Magerkurth, "Enterprise Integration of Smart Objects using Semantic Service Descriptions," in Proc. of IEEE Wireless Communication and Networking Conference (WCNC 2014), Istanbul, Turkey, 2014.

[8] Z. Shelby, K. Hartke, C. Bormann, and B. Frank, "Constrained Application Protocol (CoAP), Internet-Draft," 2011.

[9] U. Hunkeler, H. L. Truong, and A. Stanford-Clark, "MQTT-SA publish/subscribe protocol for Wireless Sensor Networks," in Communication Systems Software and Middleware and Workshops, 2008. COMSWARE 2008. 3rd International Conference on. IEEE, 2008, pp. 791798.

[10] P. Fremantle, S. Weerawarana, and R. Khalaf, "Enterprise services," Communications of the ACM, vol. 45, no. 10, pp. 77-82, 2002.

[11] J. Case, M. Fedor, M. Schoffstall, and C. Davin, A simple network management protocol (SNMP). Network Information Center, SRI International, 1989.

[12] U. Warrier, L. Besaw, L. LaBarre, and B. Handspicker, "Common management information services and protocols for the internet (CMOT and CMIP)," Request for Comments, vol. 1189, 1990.

[13] S. Kuryla and J. Schönwälder, "Evaluation of the resource requirements of SNMP agents on constrained devices," in Managing the Dynamics of Networks and Services. Springer, 2011, pp. 100-111.

[14] J. Schönwälder, H. Mukhtar, S. Joo, and K. Kim, "SNMP optimizations for constrained devices," IETF draft draft-hamid-6lowpansnmpoptimizations-03. txt, IETF, 2010.

[15] A. Sehgal, V. Perelman, S. Kuryla, and J. Schonwalder, "Management of resource constrained devices in the internet of things," Communications Magazine, IEEE, vol. 50, no. 12, pp. 144-149, 2012.

[16] O. Mazhelis, M. Waldburger, G. S. Machado, B. Stiller, and P. Tyrväinen, "Retrieving monitoring and accounting information from constrained devices in internet-of-things applications," in Emerging Management Mechanisms for the Future Internet. Springer, 2013, pp. 136-147. 